\title{
A successful case of pediatric tracheal tube exchange utilizing gum-elastic bougie and videolaryngoscope
}

\author{
Fumihiro Ohchi, Nobuyasu Komasawa*, Noriko Kadono and Toshiaki Minami \\ Department of Anesthesiology, Osaka Medical College, Osaka, Japan
}

Received 29 July 2014

Revised 4 August 2014

Accepted 15 August 2014

Here we report on our experiences with successful pediatric tracheal tube exchange utilizing the gum-elastic bougie (GEB; SmithMedical, USA) and videolaryngoscope (Pentax-AWS Airwayscope ${ }^{\circledR}$; Hoya, Japan) with an infant-size Intlock in a ventilated child, whose condition was complicated by pneumonia and dilated cardiomyopathy.

A 2-year-old female (height $77 \mathrm{~cm}$; weight $8.8 \mathrm{~kg}$ ) was transferred to our emergency department due to severe dyspnea and vital sign collapse. She was diagnosed with cardiac failure due to dilated cardiomyopathy associated with pneumonia. We performed emergent tracheal intubation with a tracheal tube with an internal diameter (ID) of $3.5 \mathrm{~mm}$, and sedated her with midazolam and dexmedetomidine. Intensive care was continued with antibiotics and mechanical ventilation, and catecholamine support with dobutamine was added. Demands for secretion management and ventilator weaning dictated tracheal tube exchange with a larger size tube. To avoid cardiopulmonary collapse associated with tube exchange or prolonged hypoxia, we decided to perform tracheal tube exchange with

\footnotetext{
*Corresponding author: Nobuyasu Komasawa, $\mathrm{MD}, \mathrm{PhD}$, Department of Anesthesiology, Osaka Medical College, Daigakumachi 2-7, Takatsuki, Osaka 569-8686, Japan. Tel.: +81 72683 2368; Fax: +81 72684 6552; E-mail: ane078@ poh.osaka-med.ac.jp.
}

AWS and GEB. First, we inserted an infant-size Intlock into the AWS with infant-size Introck and visualized the glottis. Then, we placed the GEB into the trachea through the tracheal tube. Following this, we attempted to exchange the tracheal tube with a tube of $4.5 \mathrm{~mm}$ ID, but were unable to do so. However, intubation was successful with a $4.0 \mathrm{~mm}$ ID tube. During this procedure, we observed no changes in vital signs including oxygenation.

This particular individual's condition was complicated by severe cardiopulmonary collapse associated with dilated myocardiopathy, and tracheal tube exchange required minimum interruption of oxygenation and stress by laryngoscopy. GEB is usually utilized for difficult laryngoscopy [1]. Furthermore, there is a report of successful tube exchange utilizing GEB in adult case [2]. In this case, use of the GEB allowed us to exchange the pediatric tracheal tube without leading to oxygenation failure. Evidence from pediatric cases increasingly indicates that the videolarygoscope such as AWS is suitable for difficult airway management and emergent situations [3]. The new infant-sized Intlock can fit with internal diameter tubing $3.5 \mathrm{~mm}$ or less and provide good laryngeal view [4]. In addition, laryngoscopy with AWS allowed for an improved laryngeal view with minimal change in vital signs [5]. 


\section{References}

[1] Semjen F, Bordes M, Cros AM. Intubation of infants with Pierre Robin syndrome: the use of the paraglossal approach combined with a gum-elastic bougie in six consecutive cases. Anaesthesia 2008;63(2):147-50

[2] Kishii M, Asai T, Nagata A, Shingu K. Use of the Pentax-AWS videolaryngoscope for bougie-assisted exchange of tracheal tubes. Masui 2009;58(6):765-7 (in Japanese).

[3] Lee JH, Park YH, Byon HJ, Han WK, Kim HS, Kim CS, et al. A comparative trial of the GlideScope $(\mathrm{R})$ video laryngoscope to direct laryngoscope in children with difficult direct laryngoscopy and an evaluation of the effect of blade size. Anesth Analg 2013;117(1):176-81
[4] Komasawa N, Ueki R, Yamamoto N, Nishi S, Kaminoh Y Tashiro C. Comparison of Pentax-AWS Airwayscope, Airtraq and Miller laryngoscope for tracheal intubation by novice doctors during infant cardiopulmonary resuscitation simulation: A randomized crossover trial. J Anesth 2013;27(5): 778-80.

[5] Nishikawa K, Matsuoka H, Saito S. Tracheal intubation with the PENTAX-AWS (airway scope) reduces changes of hemodynamic responses and bispectral index scores compared with the Macintosh laryngoscope. J Neurosurg Anesthesiol 2009;21(4):292-6. 